\title{
Simplified Model of Low CyCLE FATIGUe FOR RC Frames
}

\section{Discussion by Alberto Carnicero, Ricardo Perera, and Enrique Alarcón}

To model strength degradation due to low cycle fatigue, at least three different approaches can be considered. One possibility is based on the formulation of a new free energy function and damage energy release rate, as was proposed by $\mathbf{J u}$ (1989).

The second approach uses the notion of bounding surface introduced in cyclic plasticity by Dafalias and Popov (1975). From this concept, some models have been proposed to quantify damage in concrete or RC (Suaris et al. 1990). The model proposed by the author to include fatigue effects is based essentially in Marigo (1985) and can be included in this approach. In the formulation of the fatigue law, the loading-unloading irreversibility concept is employed. However, in this model, an additional constant $\alpha$ is introduced. The physical meaning of this constant is not clear, and neither is its evaluation.

In the approach developed by the discussers, the classical concepts of yield and damage domains are used and the traditional notion of damage energy release rate as a variable related to the elastic strain energy is kept.

For it, the dissipative potentials proposed by the author (1995) are slightly modified to introduce cumulative effects. The discussers propose the following function:

$$
g=Y-\left[Y_{c r}+Z(D) \xi(\omega)\right] \leq 0
$$

for the damage dissipative potential. In order to keep the coupling between the damage and the plastic domain, a modification of the plastic dissipative potential is also required:

$$
f=|M-X(D)|-M_{y}-R(D) \sqrt{\xi(\omega)} \leq 0
$$

where $\omega$ is a cumulative parameter.

The fatigue function, $\xi(\omega)$, must satisfy two restrictions:

$$
\begin{aligned}
& \xi(\omega)=1 \Leftrightarrow \omega \leq \omega_{\min } \\
& \xi(\omega)=0 \Leftrightarrow \omega=\omega_{\max }
\end{aligned}
$$

This new term introduces a softening effect in the two functions in order to include the fatigue effects.

The discussers propose as fatigue function

$$
\xi\left(\tilde{\theta}, \theta_{t}\right)=1-\left[\frac{\tilde{\theta}}{N_{f}\left(\theta_{i}\right) \cdot \theta_{t}}\right]^{1 / \mu}=1-\left[\frac{n}{N_{f}\left(\theta_{t}\right)}\right]^{1 / \mu}
$$

where $\tilde{\theta}$ and $\theta_{t}$ represent the total cumulative rotation and the total rotation (semiamplitude loop), respectively; and $N_{f}\left(\theta_{t}\right)=$ number of cycles to failure at $\theta_{t}$ semiamplitude. Therefore, the relation between $\tilde{\theta}$ and $\theta_{t}$ is a measure of the number of cycles, $n$. The most important influence of the higher cycles over the lower ones is considered across the ductility $\mu$. 
Apparently, this model introduces a new parameter, $N_{f}$. However, this parameter can be identified physically following Mander et al. (1994) or Koh and Stephen (1991); both of them gave relationships between $N_{f}$ and the deformation of the longitudinal reinforcement, but whereas the first one uses the plastic amplitude, the second uses the total amplitude of the loop. Writing these two expressions in terms of plastic rotation, we have

$$
N_{f}=2\left(\frac{0.16}{\phi_{p}} \frac{l_{p}}{d}\right)^{2}
$$

for the relationship given by Mander et al. and

$$
N_{f}=\frac{1}{2}\left(\frac{0.16}{d\left(\frac{M_{y}}{E I}+\frac{\theta_{p}}{l_{p}}\right)}\right)^{3}
$$

for the relationship given by Koh and Stephen. In both cases, the plastic hinge length, $l_{p}$, must be calculated. In the previous equations $\theta_{p}=$ plastic rotation; $d=$ distance between the reinforcement; $M_{y}=$ yield moment; $E=$ elastic modulus; and $I$ $=$ moment of inertia. The correlation between numerical and experimental results is, on balance, better using the second expression.

Two examples are presented to show the performance of

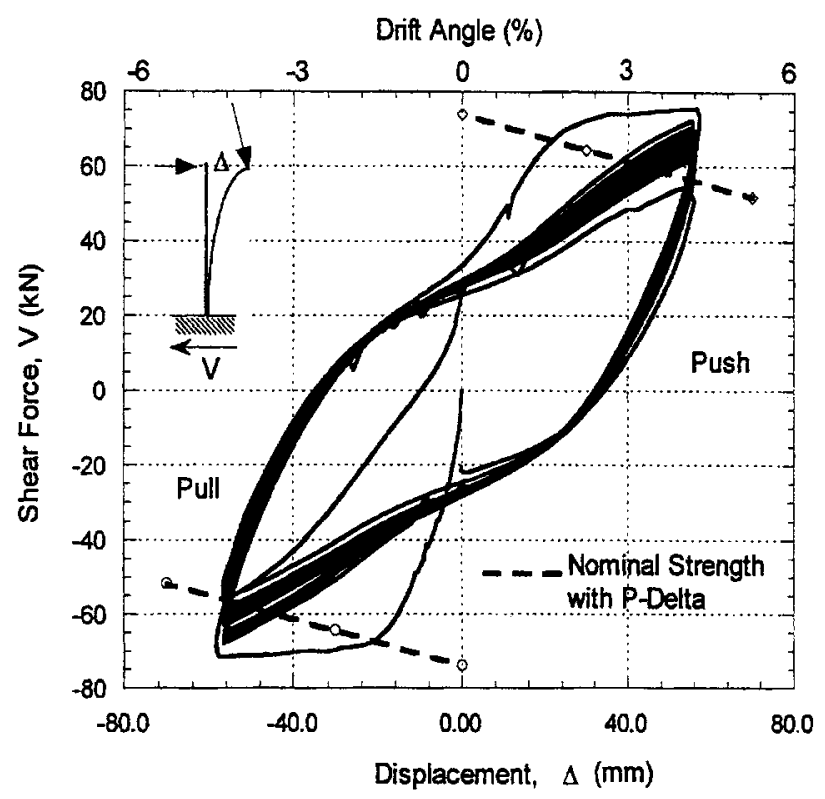

FIG. 6. Test Results Reported in Kunnath et al. (1997)

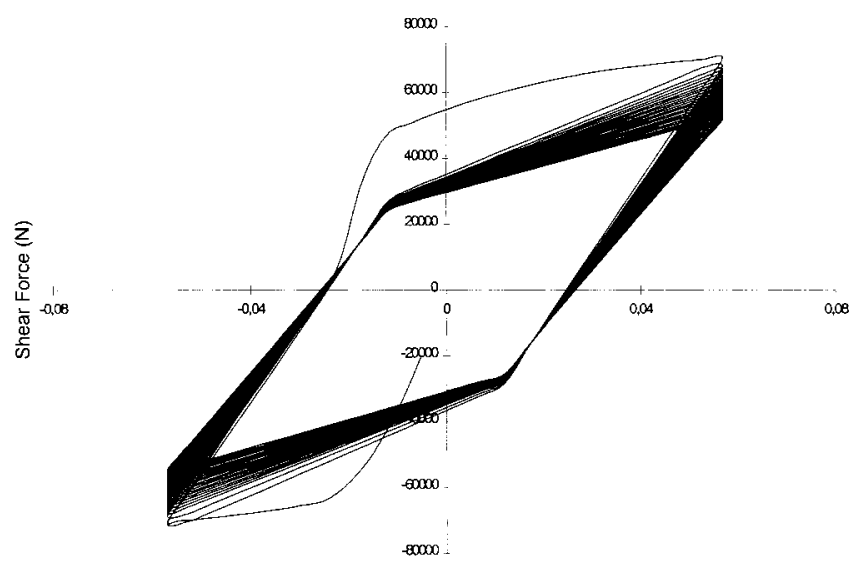

Displacerrent (m)

FIG. 6. Numerical Simulation of Test Reported in Kunnath et al. (1997)

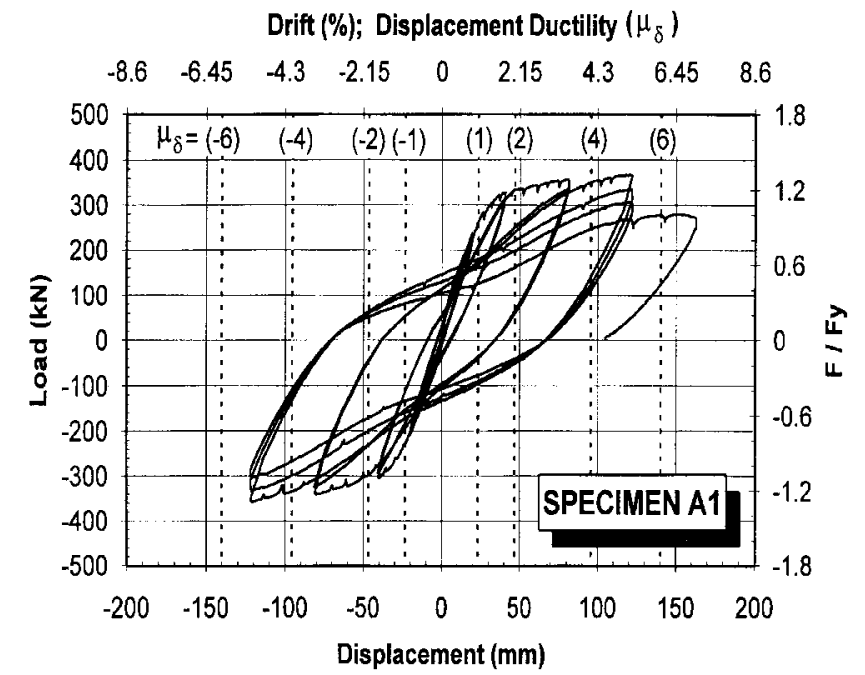

FIG. 7. Test Results Reported in Weh be et al. (1994)

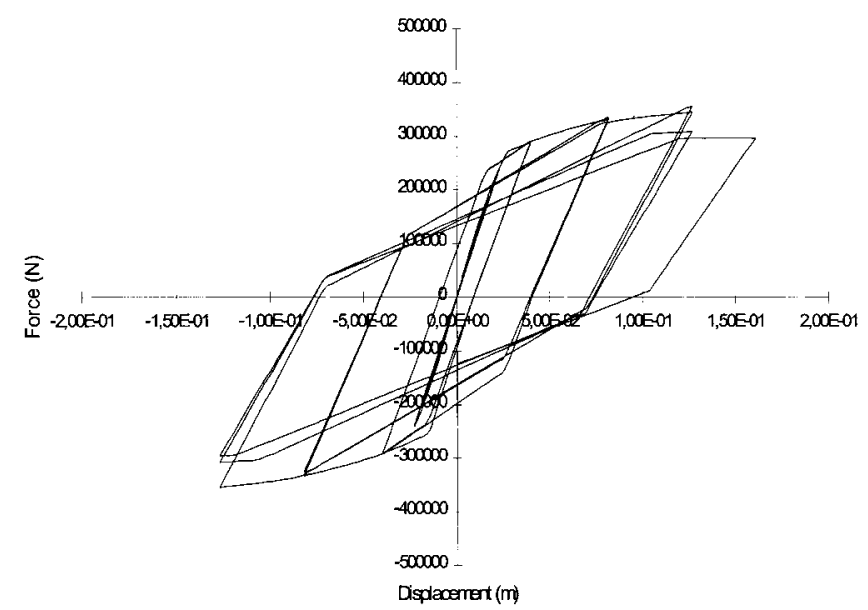

FIG. 8. Numerical Simulation of Test Reported in Wehbe et al. (1994)

this approach. Fig. 5 represents the experimental results of a circular cross-section reinforced concrete column tested by Kunnath et al. (1997), which is subjected to a constant axial load of $806 \mathrm{kN}$ and a cyclic lateral displacement of constant amplitude. The numerical simulation, shown in Fig. 6, was performed with the following parameters: $E I / L=2.51 E+7$ $\mathrm{Nm} ; M_{c r}^{+}=M_{c r}^{-}=27.420 \mathrm{kNm} ; M_{p}^{+}=87.808 \mathrm{kNm} ; M_{u}^{+}=$ $M_{u}^{-}=98.784 \mathrm{kNm} ; \theta_{p u}^{+}=\theta_{p u}^{-}=0.029 ; \alpha^{+}=\alpha^{-}=1$.

Figs. 7 and 8 show experimental and numerical results of a rectangular cross-section reinforced concrete column with moderate confinement tested by Wehbe et al. (1994). As in the previous case, the column is subjected to a constant axial load of $641 \mathrm{kN}$ and the lateral displacement is controlled. The numerical simulation has been done using the following parameters: $E I / L=2.21 E+7 \mathrm{Nm} ; M_{c r}^{+}=M_{c r}^{-}=210 \mathrm{kNm} ; M_{p}^{+}=$ $M_{p}^{-}=643 \mathrm{kNm} ; M_{u}^{+}=M_{u}^{-}=850 \mathrm{kNm} ; \theta_{p u}^{+}=\theta_{p u}^{-}=0.05 ; \alpha^{+}$ $=\alpha^{-}=1$. 